\title{
Effect of antibiotic treatment and gamma- irradiation on cuticular hydrocarbon profiles and mate choice in tsetse flies (Glossina m. morsitans)
}

Tobias Engl $1^{1,2^{*}}$, Veronika Michalkova ${ }^{3,4,5}$, Brian L. Weiss ${ }^{3}$, Güler D. Uzel ${ }^{6,7}$, Peter Takac ${ }^{4}$, Wolfgang J. Miller ${ }^{8}$, Adly M. M. Abd-Alla ${ }^{6}$, Serap Aksoy ${ }^{3}$ and Martin Kaltenpoth ${ }^{1,2^{*}}$

\begin{abstract}
Background: Symbiotic microbes represent a driving force of evolutionary innovation by conferring novel ecological traits to their hosts. Many insects are associated with microbial symbionts that contribute to their host's nutrition, digestion, detoxification, reproduction, immune homeostasis, and defense. In addition, recent studies suggest a microbial involvement in chemical communication and mating behavior, which can ultimately impact reproductive isolation and, hence, speciation. Here we investigated whether a disruption of the microbiota through antibiotic treatment or irradiation affects cuticular hydrocarbon profiles, and possibly mate choice behavior in the tsetse fly, Glossina morsitans morsitans. Four independent experiments that differentially knock down the multiple bacterial symbionts of tsetse flies were conducted by subjecting tsetse flies to ampicillin, tetracycline, or gammairradiation and analyzing their cuticular hydrocarbon profiles in comparison to untreated controls by gas chromatography - mass spectrometry. In two of the antibiotic experiments, flies were mass-reared, while individual rearing was done for the third experiment to avoid possible chemical cross-contamination between individual flies.

Results: All three antibiotic experiments yielded significant effects of antibiotic treatment (particularly tetracycline) on cuticular hydrocarbon profiles in both female and male G. m. morsitans, while irradiation itself had no effect on the CHC profiles. Importantly, tetracycline treatment reduced relative amounts of 15,19,23-trimethylheptatriacontane, a known compound of the female contact sex pheromone, in two of the three experiments, suggesting a possible implication of microbiota disturbance on mate choice decisions. Concordantly, both female and male flies preferred non-treated over tetracycline-treated flies in direct choice assays.

Conclusions: While we cannot exclude the possibility that antibiotic treatment had a directly detrimental effect on fly vigor as we are unable to recolonize antibiotic treated flies with individual symbiont taxa, our results are consistent with an effect of the microbiota, particularly the obligate nutritional endosymbiont Wigglesworthia, on $\mathrm{CHC}$ profiles and mate choice behavior. These findings highlight the importance of considering host-microbiota interactions when studying chemical communication and mate choice in insects.
\end{abstract}

Keywords: Glossina morsitans, Tsetse, Endosymbiont, Wigglesworthia, Cuticular hydrocarbons, Mate choice

\footnotetext{
*Correspondence: tengl@uni-mainz.de; mkaltenpoth@uni-mainz.de

'Insect Symbiosis Research Group, Max Planck Institute for Chemical Ecology,

Jena, Germany

Full list of author information is available at the end of the article
}

(c) International Atomic Energy Agency; licensee BioMed Central Ltd. 2018 This is an open access article distributed under the terms of the Creative Commons Attribution IGO License (https://creativecommons.org/licenses/by/3.0/igo/) which permits unrestricted use, distribution, and reproduction in any medium, provided appropriate credit to the original author(s) and the source is given. 


\section{Background}

Cuticular hydrocarbons (CHCs) are ubiquitous and both structurally and functionally diverse in insects [1]. Although the primary function of $\mathrm{CHCs}$ is the protection of the insect from water loss, they have secondarily adopted a multitude of functions in intra- and interspecific communication in a solitary as well as social context [1-5]. In particular, CHCs play an important role in mate attraction, species and sex recognition, courtship, and mate choice in many insect species $[1,6,7]$.

Most insects are associated with obligate and/or facultative microbial symbionts that can affect physiology, ecology, and evolution of their hosts in a multitude of ways [8-10], including direct or indirect effects on chemical communication and mate choice [11]. Notably, experiments in locusts revealed a direct contribution of microbial gut symbionts to the production of the host's cohesion pheromone [12, 13], and studies in fruit flies suggested that members of the microbiota can alter the $\mathrm{CHC}$ profile of the host and thereby affect mate choice decisions under certain circumstances [14-16]. Such pheromonal changes may constitute the first steps towards premating isolation and hence initiate speciation processes $[11,17]$.

Tsetse flies (Glossina spp., Diptera, Glossinidae) are associated with a taxonomically diverse microbial community. These microbes include environmentally acquired gut-associated microbes [18-20] as well as two bacterial symbionts (obligate mutualistic Wigglesworthia glossinidia and commensal Sodalis glossinidius) that are transmitted from pregnant females to their intrauterine larval offspring via maternal milk gland secretions [21, 22]. Some tsetse flies also house the reproductive symbiont Wolbachia [maternally transmitted through the germ line; 22] as well as viral and protozoan pathogens [23]. Wigglesworthia is an intracellular mutualist that serves important functions in tsetse, including supplementation of B-complex vitamins absent from vertebrate blood [24], and actuation of the development of tsetse's immune system [25, 26]. While Sodalis is consistently present in flies, its function is not yet well established. Wolbachia is less prevalent, but is known to affect host reproduction across a wide range of insect hosts, including tsetse flies, where it causes cytoplasmic incompatibility [27-29].

While the effects of the microbial symbionts on tsetse fly metabolism and reproduction have been studied in detail, their possible impact on chemical communication and mate choice remains unknown. The $\mathrm{CHCs}$ of tsetse flies are characterized by a sex-specific blend of mono-, di-, and tri-methyl alkanes $[30,31]$. Some of the long-chain methyl-branched $\mathrm{CHCs}$ have been implicated in eliciting sexual behavior of males upon contact with the females [32-36]. In G. m. morsitans, male contact with female-produced 15,19,23-trimethyl-heptatriacontane is necessary and sufficient to trigger male sexual behavior, provided that the compound is presented on a fly-like visual stimulus [32]. However, it remains elusive whether male $\mathrm{CHCs}$ also play a role for female mate choice decisions in tsetse.

Here, we set out to investigate the impact of bacterial symbionts on CHC profiles of Glossina morsitans morsitans and their possible influence on sexual selection and mating success. We used gas chromatography coupled to mass spectrometry (GC-MS) to analyze CHC profiles [37] of tsetse flies after antibiotic- as well as irradiation-mediated perturbations of the host-symbiont equilibrium [27, 38]. In addition, we assessed the effect of antibiotics on mating success of male and female G. m. morsitans.

\section{Methods}

\section{Sampling and treatments}

Glossina morsitans morsitans for antibiotic treatments were reared on bovine blood (Hemostat laboratories, Dixon, CA) in the laboratory at Yale University at $24{ }^{\circ} \mathrm{C}$ and on a $14 \mathrm{~h} / 10 \mathrm{~h}$ light/dark photoregime. Two fly treatment groups were established by feeding pregnant females a diet supplemented with either ampicillin (Amp; $50 \mu \mathrm{g} / \mathrm{ml}$ blood; Pais et al., 2008) or tetracycline (Tet; $25 \mu \mathrm{g} / \mathrm{ml}$ blood; Alam et al., 2011). Tet-treated females were also supplemented with yeast extract $[1 \% w / v ; 24]$ to partially restore reproductive sterility that occurs in the absence of obligate Wigglesworthia [38]. Amp treatment of pregnant tsetse flies eliminates only Wigglesworthia from milk secretions such that larval offspring undergo their entire developmental program in the absence of this obligate symbiont but in the presence of Sodalis and Wolbachia [38]. Tet treatment eliminates all bacteria from pregnant females so that larvae undergo their entire developmental program in the absence of all bacteria [27]. Offspring from Amp and Tet treated mothers, which were used to test the impact of symbiont titer knockdown on tsetse's $\mathrm{CHC}$ profile, are hereafter designated $\mathrm{Gmm}^{\mathrm{Wgm}-}$ and $\mathrm{Gmm}^{\mathrm{Apo}}$ (Apo = aposymbiotic), respectively. Gmm ${ }^{\text {Wgm- }}$ and $G m m^{\text {Apo }}$ flies were reared either collectively (experiment 1 and 2) or individually (experiment 3) on antibiotic-free bovine blood and sampled for chemical analyses. For experiment 3, only control and $\mathrm{Gmm}^{\mathrm{Apo}}$ individuals were generated because rearing tsetse individually is untenable on a large scale. For each treatment group, 10 unmated male and 10 virgin female flies were sampled at day 10 (experiments $1+3$ ) or day 5 (experiment 2) after adult emergence.

Glossina morsitans morsitans flies used for irradiation treatments were originally from Zimbabwe and maintained since 1997 at the Insect Pest Control Laboratory (IPCL) of the Joint FAO/IAEA Division of Nuclear Techniques in Food and Agriculture, Seibersdorf, Austria. Tsetse flies were maintained at a temperature of $23 \pm 1{ }^{\circ} \mathrm{C}$, a relative 
humidity of $75-80 \%$ under on a $12 \mathrm{~h} / 12 \mathrm{~h}$ light/dark photoregime. Experimental flies were fed on defibrinated bovine blood using an artificial (in vitro) membrane feeding system for 15-20 min three times per week [39]. Male flies were either irradiated as 22-day old pupae (early), at the very late pupal stage at which females had already emerged (29-days old (late)), or as 5-day old adults (adult). The irradiation treatment was performed using a Gammacell 220 60 Co irradiator (Nordion Ltd., Ottawa, Canada) by exposing the samples for different time periods to receive an irradiation dose of 110,50 or 20 Gy. Non irradiated flies were used as a control (0 Gy). Twenty two-day and 29-day old pupae were irradiated in a $9 \mathrm{~cm}$ diameter petri-dish while the 5-day old males were irradiated in individual small cages $(4 \mathrm{~cm}$ diameter $\times 6 \mathrm{~cm}$ high) (one male/cage). Irradiated pupae were separated and reared individually in a pill sorter until emergence. After emergence each male was individually placed in a small cage and maintained until day 10 after emergence. Depending on the time and dosage, irradiation treatment has variable effects on Sodalis and Wolbachia, but not Wigglesworthia titers [40]. Specifically, in adult flies emerging from early irradiated (22-day old) pupae, Sodalis density was decreased at 24 h post emergence and recovered over time until day 14 post eclosure, while the Wigglesworthia titer did not differ between treatment and control groups, and Wolbachia density was increased at emergence but decreased again over time. In the males emerging from late irradiated (29-day old) pupae, both Sodalis and Wolbachia density was reduced during the first week after emergence and then recovered over time. In males irradiated as adults, Sodalis density decreased after irradiation while the Wolbachia density increased at $24 \mathrm{~h}$ post irradiation and then decreased again over time.

\section{Extraction of samples and GC-MS analysis}

Individual flies were extracted in hexane. $2 \mu \mathrm{g}$ of heneicosane was added as internal standard to allow for later quantification of hydrocarbons. Extracts were evaporated to about 20-30 $\mu \mathrm{l}$ of hexane under a constant stream of

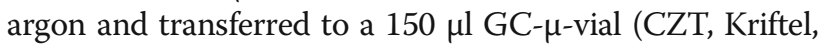
Germany) for Gaschrommatography-Massspectrometry analysis. An aliquot of $1 \mu \mathrm{l}$ of each sample was injected into a Varian 450GC gas chromatograph coupled to a Varian 240MS mass spectrometer (Agilent Technologies, Böblingen, Germany) using a split/splitless injector at $250{ }^{\circ} \mathrm{C}$ with the purge valve opened after $60 \mathrm{~s}$. The GC was equipped with a DB5-MS capillary column $(30 \mathrm{~m} \times$ $0.25 \mathrm{~mm}$ diameter, film thickness: $0.25 \mu \mathrm{m}$, Agilent Technologies) and programmed from 150 to $300{ }^{\circ} \mathrm{C}$ at $15{ }^{\circ} \mathrm{C} /$ min with a $27 \mathrm{~min}$. final isothermal hold. Helium was used as carrier gas, with a constant flow rate of $1 \mathrm{ml} / \mathrm{min}$. Mass spectra were recorded using electron impact ionization (EI-MS). Data acquisition and quantifications were achieved with MS Workstation Version 6.9.3 Software (Agilent Technologies). The peaks were identified by their mass spectra in comparison to previously published analyses of G. m. morsitans cuticular hydrocarbon profiles [30]. Peak areas were automatically integrated using the MS Workstation Software. Finally, the success of this integration was controlled manually for every peak. Some substances had to be combined for the analysis, as the peaks were not always clearly separated in the chromatograms.

\section{Mate choice assays}

Individual control males or females were given a simultaneous choice between one control and one $G m m^{\text {Apo }}$ mate, respectively. All flies were 5 days old adults. To later distinguish the individuals, the last tarsal segment was cut from either the right or left mid leg. The control and $\mathrm{Gmm}^{\text {Apo }}$ mate were set up in the clean round colony cage with $20 \mathrm{~cm}$ of diameter and height of $5 \mathrm{~cm}, 1$ day post feeding and $6 \mathrm{~h}$ before the actual experiment. An individual control male/female was inserted into the middle of the cage while the potential mates were held on the opposite side of cage by shading them with a black blanket. After the removal of the blanket, the control fly was given the ability to come into contact with both potential mates before choosing a mating partner. Matings were scored visually by observing the cage for $3 \mathrm{~h}$ or until the end of a successful mating, which lasts in G. m. morsitans 2 h [41]. For male choice, 30 replicates were performed, while 17 replicates were done for female choice assays due to the availability of flies.

\section{Statistical analysis}

Since CHC profiles of tsetse flies are sex-specific, the profiles of males and females were analyzed separately. To compare absolute amounts of hydrocarbons across treatment groups, the total amount of all compounds (combined) was calculated from the combined peak areas by comparison to the peak area of the internal standard $(=2 \mu \mathrm{g})$. For the known contact sex pheromone of female G. m. morsitans, 15,19,23-trimethyl-heptatriacontane [32], absolute and relative amounts were calculated for each individual, based on the internal standard and the total peak area of all hydrocarbons, respectively. The resulting values were compared among antibiotic treatment groups by ANOVA with Tukey post-hoc comparisons. Irradiation treatment groups were analyzed in a two-factorial ANOVA to test for effects of the dosage and age/developmental stage at which the flies were subjected to irradiation.

For all other analyses, relative amounts were calculated from the peak areas and then log-ratio-transformed according to Aitchison [42]. In order to test for differences in chemical profiles across groups, principal component 
analyses (PCAs) were performed to reduce the number of variables, and the resulting PCs (with Eigenvalues $>0.9$ ) were used for discriminant analyses (DAs) to test for among-group differences. Chi-squared tests were performed for the mate choice assays. All statistical analyses were done with SPSS 17.0.

\section{Results}

\section{CHC composition in G. m. Morsitans}

As described earlier [30], CHC profiles of G. m. morsitans were dominated by mono-, di-, and tri-methyl alkanes, and there were distinct sex-specific differences, with females generally showing more compounds with longer carbon backbones (Tables 1, 2 and 3). The main components of female CHC profiles were 2-methyl-triacontane, 15,19- and 17,21-dimethyl-heptatriacontane, and 15,19,23-trimethyl-heptatriacontane, which together accounted for about $70 \%$ of the complete CHCs in control flies (Table 1). In males, 2-methyl-triacontane and 11,15-dimethyl-tritriacontane dominated, amounting to about $40 \%$ of the total $\mathrm{CHC}$ profile in control flies from Vienna and $70 \%$ in control flies from Yale (Tables 2, 3). In addition to these differences in the dominant compounds, males reared in Vienna showed slightly more of the longer carbon backbone compounds then males reared at Yale (Tables 2, 3).

\section{Influence of antibiotic treatment on $\mathrm{CHC}$ profiles in mass- reared female flies}

Antibiotic treatment had no effect on the total amount of CHCs in 10-day-old females (Fig. 1a; ANOVA, $\mathrm{F}_{2,27}=1.154$, $p=0.330)$. $\mathrm{Gmm}^{\text {Apo }}$ females showed a non-significant tendency towards lower absolute amounts of 15,19,23trimethyl-heptatriacontane (Fig. 1c; ANOVA, $\mathrm{F}_{2,27}=$ 1.267, $p=0.298$ ). A comparison of the relative amounts of 15,19,23-trimethyl-heptatriacontane revealed significantly lower proportions of sex pheromone in $\mathrm{Gmm}^{\mathrm{Apo}}$ females as compared to control and $\mathrm{Gmm}^{\mathrm{Wgm}-}$ flies (Fig. 1d; ANOVA, $\mathrm{F}_{2,27}=6.291, p=0.006$ ).

Based on the 19 quantified peaks, four principal components were extracted, capturing $83.9 \%$ of the total variance. A discriminant analysis (DA) based on the four PCs including all three treatment groups yielded a significant difference in CHC profiles across groups (Fig. 2a; Wilks' Lambda = $\left.0.354, \mathrm{X}^{2}=26.5, \mathrm{df}=8, p=0.001\right)$. Based on the two discriminant functions, $60 \%$ of the cases were correctly classified (30\% would be expected by chance). Subsequent DAs of pairwise combinations of the three groups revealed no

Table $1 \mathrm{CHC}$ profiles of 5- and 10-day old antibiotic-treated ( $\mathrm{Gmm}^{\mathrm{Wgm}-}=$ ampicillin; $\mathrm{Gmm}^{\mathrm{Apo}}=$ tetracycline) and control female $\mathrm{G}$. $m$. morsitans after mass- or individual rearing. Compounds are sorted by class (mono-, di-, and trimethyl-alkanes). Given are average relative amounts of CHCs (in percent) $+/$ - standard deviation, as well as the total absolute amount of CHCs as determined by comparison with an internal standard. Me = methyl

\begin{tabular}{|c|c|c|c|c|c|c|c|c|}
\hline \multirow[t]{2}{*}{ Compound } & \multicolumn{3}{|c|}{ 5-day old, mass-rearing } & \multicolumn{3}{|c|}{ 10-day old, mass-rearing } & \multicolumn{2}{|c|}{ 10-day old, individual rearing } \\
\hline & Control & $\mathrm{Gmm}^{\mathrm{Wgm}-}$ & $G m m^{A p o}$ & Control & $\mathrm{Gmm}^{\text {Wgm- }}$ & $G m m^{\text {Apo }}$ & Control & $G m m^{\mathrm{Apo}}$ \\
\hline 2Me-C28 & $0.41 \pm 0.13$ & $0.58 \pm 0.19$ & $0.83 \pm 0.62$ & $0.28 \pm 0.11$ & $0.80 \pm 0.70$ & $0.75 \pm 0.40$ & $0.46 \pm 0.14$ & $1.16 \pm 0.32$ \\
\hline $2 \mathrm{Me}-\mathrm{C} 29$ & $0.77 \pm 0.23$ & $1.81 \pm 0.60$ & $1.32 \pm 0.76$ & $1.26 \pm 0.42$ & $1.78 \pm 0.70$ & $1.89 \pm 0.45$ & $1.88 \pm 0.70$ & $3.56 \pm 0.61$ \\
\hline 2Me-C30 & $13.16 \pm 2.71$ & $21.57 \pm 6.70$ & $13.89 \pm 5.51$ & $20.72 \pm 4.04$ & $20.75 \pm 4.21$ & $26.15 \pm 9.91$ & $31.02 \pm 6.64$ & $37.94 \pm 3.87$ \\
\hline 2Me-C31 & $0.47 \pm 0.20$ & $0.80 \pm 0.32$ & $0.57 \pm 0.29$ & $0.63 \pm 0.16$ & $0.80 \pm 0.78$ & $0.97 \pm 0.48$ & $1.20 \pm 0.38$ & $2.19 \pm 1.00$ \\
\hline 2Me-C32 & $0.89 \pm 0.25$ & $1.55 \pm 0.50$ & $1.13 \pm 0.65$ & $0.89 \pm 0.26$ & $0.90 \pm 0.30$ & $1.12 \pm 0.36$ & $1.47 \pm 0.32$ & $2.09 \pm 0.70$ \\
\hline 2Me-C34 & $0.08 \pm 0.05$ & $0.14 \pm 0.12$ & $0.10 \pm 0.07$ & $0.06 \pm 0.04$ & $0.12 \pm 0.07$ & $0.11 \pm 0.07$ & $0.05 \pm 0.02$ & $0.12 \pm 0.08$ \\
\hline 11,15-diMe-C33 & $0.46 \pm 0.14$ & $1.04 \pm 0.32$ & $0.96 \pm 0.53$ & $0.43 \pm 0.13$ & $0.46 \pm 0.33$ & $0.99 \pm 1.23$ & $0.18 \pm 0.04$ & $0.82 \pm 0.70$ \\
\hline diMe-C34 & $0.36 \pm 0.09$ & $0.44 \pm 0.09$ & $0.45 \pm 0.14$ & $0.20 \pm 0.04$ & $0.22 \pm 0.10$ & $0.24 \pm 0.21$ & $0.32 \pm 0.07$ & $0.34 \pm 0.16$ \\
\hline 15,19-diMe-C35 & $9.13 \pm 1.31$ & $8.51 \pm 1.15$ & $8.06 \pm 1.33$ & $5.54 \pm 0.56$ & $5.42 \pm 0.08$ & $5.62 \pm 2.51$ & $7.76 \pm 0.98$ & $5.84 \pm 1.71$ \\
\hline 15,19 + 16,20-diMe-C36 & $6.85 \pm 0.54$ & $6.35 \pm 0.43$ & $7.03 \pm 0.77$ & $4.35 \pm 0.54$ & $3.74 \pm 0.45$ & $4.43 \pm 1.22$ & $5.66 \pm 0.66$ & $4.32 \pm 0.71$ \\
\hline 15,19 + 17,21-diMe-C37 & $21.80 \pm 3.82$ & $17.61 \pm 2.71$ & $17.60 \pm 2.35$ & $13.72 \pm 1.89$ & $13.30 \pm 3.84$ & $15.11 \pm 3.36$ & $13.67 \pm 2.63$ & $10.19 \pm 2.21$ \\
\hline diMe-C38 & $1.57 \pm 0.19$ & $1.47 \pm 0.24$ & $1.83 \pm 0.58$ & $0.90 \pm 0.21$ & $0.66 \pm 0.30$ & $1.06 \pm 0.23$ & $0.74 \pm 0.22$ & $0.64 \pm 0.12$ \\
\hline 13,17,21-triMe-C35 & $0.64 \pm 0.15$ & $0.51 \pm 0.08$ & $0.69 \pm 0.10$ & $0.57 \pm 0.13$ & $1.19 \pm 1.27$ & $0.42 \pm 0.14$ & $0.85 \pm 0.19$ & $0.96 \pm 0.13$ \\
\hline triMe-C36 & $1.86 \pm 0.44$ & $1.65 \pm 0.29$ & $2.17 \pm 0.33$ & $1.81 \pm 0.36$ & $1.85 \pm 0.63$ & $1.39 \pm 0.35$ & $2.16 \pm 0.53$ & $1.94 \pm 0.28$ \\
\hline 15,19,23-triMe-C37 & $27.35 \pm 3.18$ & $23.05 \pm 2.69$ & $27.85 \pm 3.55$ & $36.58 \pm 2.52$ & $37.67 \pm 6.04$ & $28.88 \pm 7.64$ & $25.10 \pm 3.29$ & $21.91 \pm 2.63$ \\
\hline 15,19,23-triMe-C38 & $8.40 \pm 1.42$ & $7.90 \pm 1.51$ & $9.93 \pm 2.06$ & $7.88 \pm 1.83$ & $6.76 \pm 1.86$ & $7.01 \pm 0.55$ & $6.52 \pm 1.82$ & $5.14 \pm 0.98$ \\
\hline 15,19,23-triMe-C39 & $5.41 \pm 1.16$ & $4.46 \pm 1.32$ & $5.24 \pm 1.58$ & $3.78 \pm 1.00$ & $2.89 \pm 0.92$ & $3.43 \pm 0.67$ & $0.24 \pm 0.15$ & $0.19 \pm 0.06$ \\
\hline unknown & $0.37 \pm 0.15$ & $0.53 \pm 0.22$ & $0.36 \pm 0.15$ & $0.41 \pm 0.19$ & $0.70 \pm 0.68$ & $0.45 \pm 0.27$ & $0.70 \pm 0.30$ & $0.63 \pm 0.20$ \\
\hline Total CHC amount $(\mu \mathrm{g})$ & $35.04 \pm 9.36$ & $29.70 \pm 26.66$ & $43.32 \pm 18.73$ & $33.43 \pm 29.28$ & $24.17 \pm 20.06$ & $18.48 \pm 14.72$ & $26.98 \pm 10.48$ & $28.22 \pm 6.44$ \\
\hline
\end{tabular}


Table 2 CHC profiles of 5- and 10-day old antibiotic-treated ( $\mathrm{Gmm}^{\mathrm{Wgm}-}=$ ampicillin; $\mathrm{Gmm}^{\text {Apo }}=$ tetracycline) and control male $\mathrm{G}$. $\mathrm{m}$. morsitans after mass- or individual rearing. Compounds are sorted by class (mono-, di-, and trimethyl-alkanes). Given are average relative amounts of $\mathrm{CHCs}$ (in percent) $+/$ - standard deviation, as well as the total absolute amount of CHCs as determined by comparison with an internal standard. Me $=$ methyl

\begin{tabular}{|c|c|c|c|c|c|c|c|c|}
\hline \multirow[t]{2}{*}{ Compound } & \multicolumn{3}{|c|}{ 5-day old, mass-rearing } & \multicolumn{3}{|c|}{ 10-day old, mass-rearing } & \multicolumn{2}{|c|}{ 10-day old, individual rearing } \\
\hline & Control & $\mathrm{Gmm}^{\text {Wgm- }}$ & $G m m^{\text {Apo }}$ & Control & $\mathrm{Gmm}^{\mathrm{Wgm}-}$ & $G m m^{\text {Apo }}$ & Control & $G m m^{\text {Apo }}$ \\
\hline 2Me-C28 & $0.42 \pm 0.18$ & $1.36 \pm 0.91$ & $1.20 \pm 0.37$ & $0.45 \pm 0.18$ & $0.84 \pm 0.42$ & $2.15 \pm 0.89$ & $0.58 \pm 0.10$ & $1.29 \pm 0.49$ \\
\hline 2Me-C29 & $4.36 \pm 1.03$ & $6.21 \pm 2.17$ & $5.74 \pm 1.00$ & $8.83 \pm 1.77$ & $8.10 \pm 3.03$ & $7.59 \pm 0.52$ & $8.80 \pm 1.11$ & $9.24 \pm 1.69$ \\
\hline 2Me-C30 & $19.52 \pm 1.67$ & $21.89 \pm 3.40$ & $21.81 \pm 1.70$ & $20.42 \pm 2.82$ & $22.96 \pm 2.01$ & $29.22 \pm 4.91$ & $22.12 \pm 2.07$ & $29.23 \pm 4.85$ \\
\hline 2Me-C31 + 9,13-diMe-C31 & $4.39 \pm 075$ & $5.59 \pm 1.70$ & $4.62 \pm 0.52$ & $3.79 \pm 0.24$ & $5.11 \pm 0.87$ & $5.03 \pm 0.87$ & $6.54 \pm 0.40$ & $7.46 \pm 1.70$ \\
\hline 2Me-C32 + diMe-C32 & $4.47 \pm 0.37$ & $5.79 \pm 0.82$ & $5.48 \pm 0.43$ & $3.32 \pm 0.35$ & $3.78 \pm 0.48$ & $4.40 \pm 0.25$ & $5.57 \pm 0.67$ & $5.73 \pm 1.32$ \\
\hline 11,15-diMe-C33 & $49.51 \pm 2.60$ & $37.92 \pm 7.16$ & $39.70 \pm 2.55$ & $51.62 \pm 3.77$ & $46.12 \pm 4.19$ & $36.18 \pm 3.99$ & $47.04 \pm 2.04$ & $36.00 \pm 9.06$ \\
\hline 12,16-diMe-C34 & $5.59 \pm 0.47$ & $5.85 \pm 1.02$ & $5.20 \pm 0.31$ & $3.30 \pm 0.47$ & $2.96 \pm 0.51$ & $3.77 \pm 0.51$ & $4.24 \pm 0.65$ & $3.53 \pm 0.60$ \\
\hline 15,19-diMe-C35 + 8-Me-C35:1 & $9.28 \pm 2.61$ & $11.70 \pm 2.03$ & $12.64 \pm 2.40$ & $5.36 \pm 0.94$ & $5.09 \pm 0.93$ & $8.17 \pm 1.53$ & $1.96 \pm 0.45$ & $2.07 \pm 0.84$ \\
\hline 7,11,15-triMe-C33 & $1.06 \pm 0.18$ & $1.45 \pm 0.37$ & $1.48 \pm 0.21$ & $0.91 \pm 0.17$ & $2.00 \pm 0.91$ & $1.27 \pm 0.22$ & $1.10 \pm 0.19$ & $1.78 \pm 0.86$ \\
\hline unknown1 & $0.11 \pm 0.08$ & $0.18 \pm 0.09$ & $0.13 \pm 0.08$ & $0.75 \pm 0.38$ & $0.10 \pm 0.09$ & $0.23 \pm 0.08$ & $0.29 \pm 0.12$ & $0.32 \pm 0.12$ \\
\hline unknown2 & $0.39 \pm 0.10$ & $0.61 \pm 0.15$ & $0.62 \pm 0.07$ & $0.37 \pm 0.07$ & $1.17 \pm 0.68$ & $0.69 \pm 0.09$ & $0.62 \pm 0.15$ & $1.56 \pm 1.11$ \\
\hline unknown3 & $0.28 \pm 0.05$ & $0.38 \pm 0.13$ & $0.38 \pm 0.04$ & $0.20 \pm 0.04$ & $0.52 \pm 0.22$ & $0.30 \pm 0.09$ & $0.37 \pm 0.10$ & $0.65 \pm 0.40$ \\
\hline unknown4 & $0.62 \pm 0.20$ & $1.07 \pm 0.32$ & $0.99 \pm 0.19$ & $0.68 \pm 0.05$ & $1.26 \pm 0.46$ & $0.99 \pm 0.11$ & $0.77 \pm 0.12$ & $1.14 \pm 0.49$ \\
\hline Total CHC amount $(\mu \mathrm{g})$ & $17.17 \pm 7.91$ & $20.95 \pm 7.10$ & $15.89 \pm 4.53$ & $25.04 \pm 12.89$ & $19.62 \pm 10.43$ & $4.30 \pm 1.82$ & $38.29 \pm 8.94$ & $36.25 \pm 19.02$ \\
\hline
\end{tabular}

significant difference between control and $\mathrm{Gmm}^{\mathrm{Wgm}-}$ flies (Wilks' Lambda $=0.595, \mathrm{X}^{2}=8.30, \mathrm{df}=4, \quad p=0.081$ ), but significant differences between control and $G m m^{\text {Apo }}$ flies (Wilks' Lambda $=0.498, \mathrm{X}^{2}=11.1, \mathrm{df}=4, p=0.025$ ) and between $\mathrm{Gmm}^{\text {Wgm- }}$ and $\mathrm{Gmm}^{\text {Apo }}$ flies (Wilks' Lambda $=0.402$, $\mathrm{X}^{2}=14.6, \mathrm{df}=4, p=0.006$ ), respectively.

In 5-day-old females, there was also no difference in total amount of CHCs across groups (Fig. 1a; ANOVA, $\mathrm{F}_{2,27}=$ 1.234, $p=0.307)$. $\mathrm{Gmm}^{\text {Wgm- }}$ females showed a non-significant tendency towards lower absolute amounts of 15,19,23-trimethyl-heptatriacontane (Fig. 1c; ANOVA, $F_{2,27}$ $=1.785, p=0.187$ ). A comparison of the relative amounts of 15,19,23-trimethyl-heptatriacontane revealed significantly lower proportions of sex pheromone in $\mathrm{Gmm}^{\mathrm{Wgm}-}$ females as compared to control and $\mathrm{Gmm}^{\text {Apo }}$ flies (Fig. 1d; ANOVA, $\mathrm{F}_{2,27}=6.981, \quad p=0.004$; Tukey HSD $p=0.014$ for control-Gmm ${ }^{W g m-}$ and $p=0.001$ for $\mathrm{Gmm}^{\text {Wgm- }}-\mathrm{Gmm}^{\mathrm{Apo}}$ ).

Based on the 18 quantified peaks, four principal components were extracted, capturing $85.0 \%$ of the total variance. A discriminant analysis (DA) based on the four PCs including all three treatment groups yielded a significant difference in $\mathrm{CHC}$ profiles across groups (Fig. 2b; Wilks' Lambda $\left.=0.224, \mathrm{X}^{2}=38.1, \mathrm{df}=8, p<0.001\right)$. Based on the two discriminant functions, $83.3 \%$ of the cases were correctly classified (30\% would be expected by chance). Subsequent DAs of pairwise combinations of the three groups revealed a significant difference between control and $\mathrm{Gmm}^{\text {Wgm- }}$ flies (Wilks' Lambda $=0.232, \mathrm{X}^{2}=23.37, \mathrm{df}=4$, $p<0.001$ ), between control and $\mathrm{Gmm}^{\text {Apo }}$ flies (Wilks' Lambda $\left.=0.367, \quad \mathrm{X}^{2}=16.0, \mathrm{df}=4, \quad p=0.003\right)$ and also between $\mathrm{Gmm}^{W g m-}$ - and $\mathrm{Gmm}^{\text {Apo }}$ flies (Wilks' Lambda = $\left.0.405, \mathrm{X}^{2}=14.5, \mathrm{df}=4, p=0.006\right)$, respectively.

Influence of antibiotic treatment on $\mathrm{CHC}$ profiles in massreared male flies

In 10-day-old male flies, control and $\mathrm{Gmm}^{\mathrm{Wgm}-}$ individuals showed on average 5-6 times higher total amounts of CHCs than did $\mathrm{Gmm}^{\text {Apo }}$ flies (Fig. 1b; ANOVA, $\mathrm{F}_{2,25}$ $=10.03, p=0.001$ ). Post-hoc comparisons (Tukey HSD) revealed these differences to be significant $(p=0.001$ and

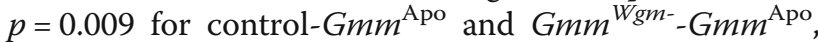
respectively), while there was no difference between control and $\mathrm{Gmm}^{W g m-}$ flies $(p=0.457)$. Based on the 13 quantified peaks, four principal components were extracted, capturing $84.3 \%$ of the total variance. A discriminant analysis (DA) based on the four PCs including all three treatment groups yielded a significant difference in $\mathrm{CHC}$ profiles across groups (Fig. 3a; Wilks' Lambda = $\left.0.046, \mathrm{X}^{2}=72.5, \mathrm{df}=8, p<0.001\right)$. Based on the two discriminant functions, $96.4 \%$ of the cases were correctly classified (30\% would be expected by chance). Subsequent DAs of all pairwise combinations of the three groups revealed significant differences between all groups: control vs. $\mathrm{Gmm}^{\mathrm{Wgm}^{-}}$: Wilks' Lambda $=0.233, \mathrm{X}^{2}=23.3$, $\mathrm{df}=4, p<0.001$; control vs. $\mathrm{Gmm}^{\mathrm{Apo}}$ : Wilks' Lambda = 0.076, $\mathrm{X}^{2}=36.0, \mathrm{df}=4, p<0.001 ; \mathrm{Gmm}^{\mathrm{Wgm-}}-$ vs. $\mathrm{Gmm}^{\mathrm{Apo}}$ : Wilks' Lambda $=0.177, \mathrm{X}^{2}=24.2, \mathrm{df}=4, p<0.001$.

Antibiotic treatment had no effect on the total amount of $\mathrm{CHCs}$ in 5-day-old males (Fig. 1b; ANOVA, $\mathrm{F}_{2,27}=$ $1.565, p=0.227$ ). Based on the 13 quantified peaks, five 


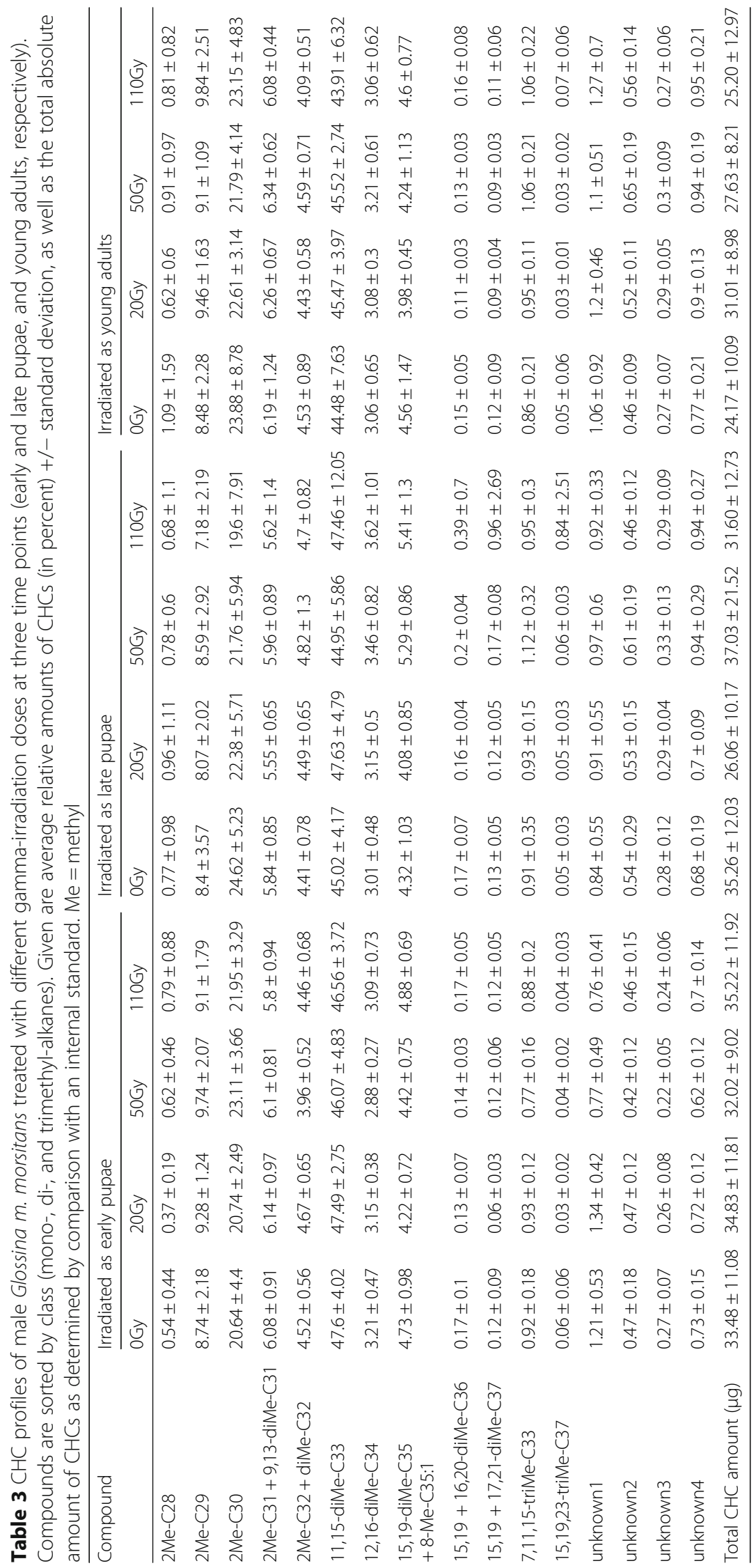



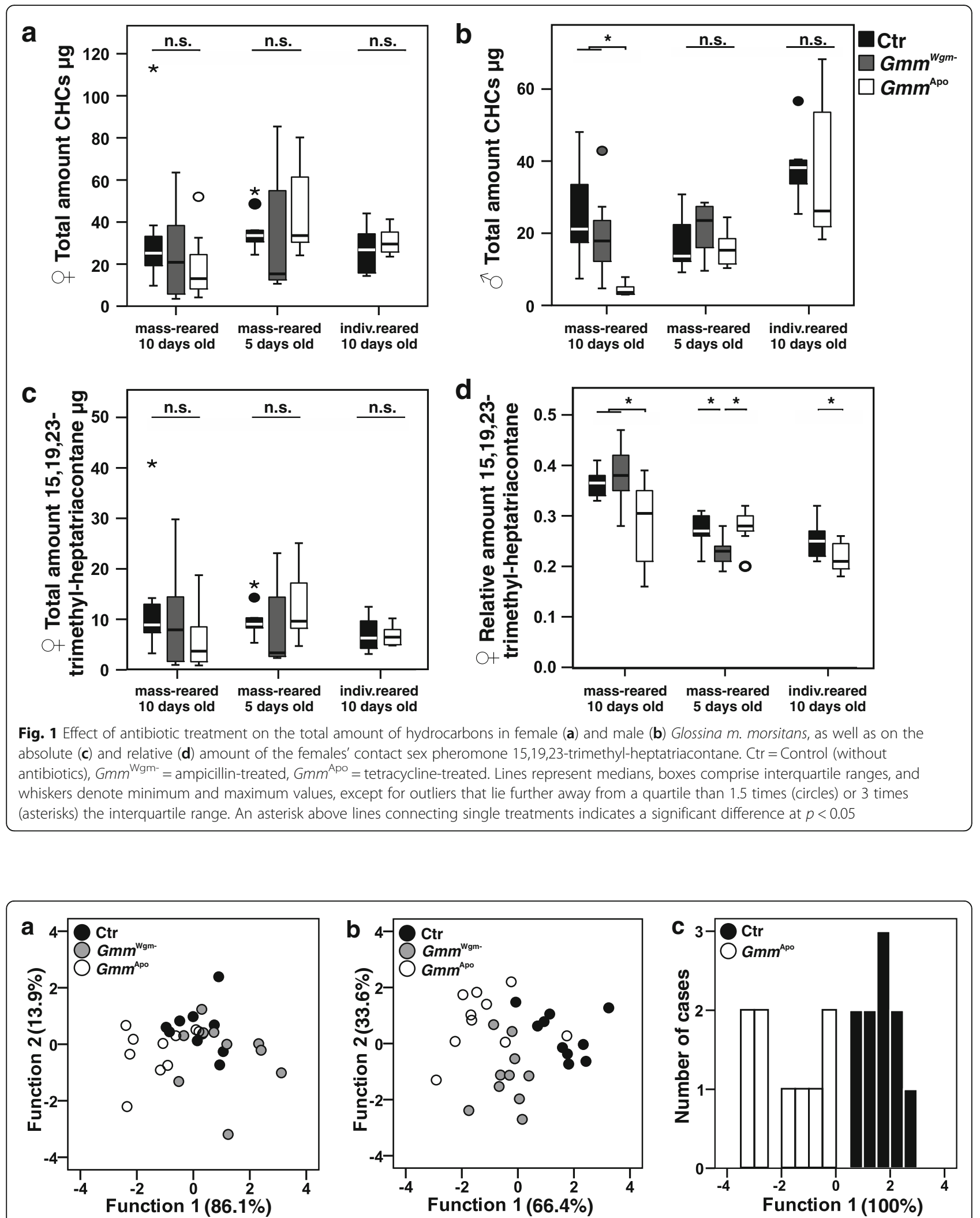

Fig. 2 Effect of antibiotic treatment on CHC profiles of female tsetse flies (G. m. morsitans). Discriminant analyses based on log-ratio transformed relative amounts of (a) CHCs of mass-reared, 10 day old females, (b) mass-reared 5 day old females, and (c) individually reared, 10 day old females. Ctr $=$ Control (without antibiotics), $\mathrm{Gmm}^{\mathrm{Wgm}-}=$ ampicillin-treated, $\mathrm{Gmm}^{\mathrm{Apo}}=$ tetracycline-treated 

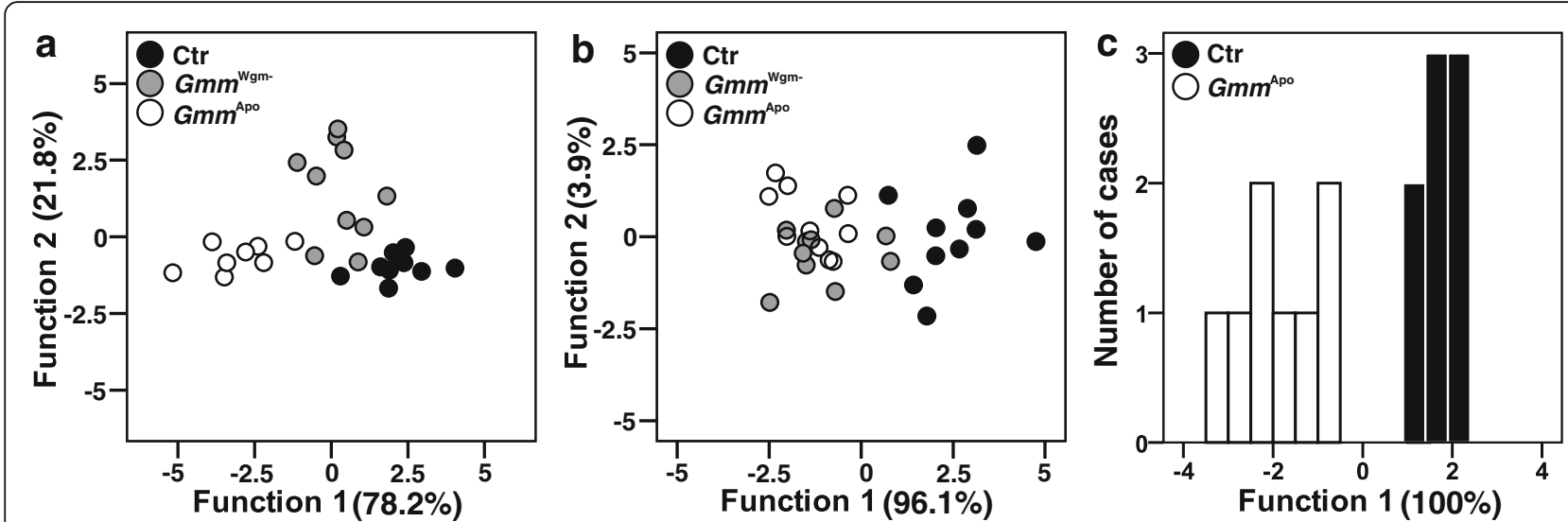

Fig. 3 Effect of antibiotic treatment on CHC profiles of male tsetse flies (G. m. morsitans). Discriminant analyses based on log-ratio transformed relative amounts of (a) CHCs of mass-reared, 10 day old males, (b) mass-reared 5 day old males, and (c) individually reared, 10 day old males. Ctr $=$ Control (without antibiotics), $\mathrm{Gmm}^{\mathrm{Wgm}-}=$ ampicillin-treated, $\mathrm{Gmm}^{\mathrm{Apo}}=$ tetracycline-treated

principal components were extracted, capturing $88.8 \%$ of the total variance. A discriminant analysis (DA) based on the four PCs including all three treatment groups yielded a significant difference in $\mathrm{CHC}$ profiles across groups (Fig. 3b; Wilks' Lambda $=0.207, \mathrm{X}^{2}=39.3, \mathrm{df}=$ $10, p<0.001)$. Based on the two discriminant functions, $70.0 \%$ of the cases were correctly classified (30\% would be expected by chance). Subsequent DAs of all pairwise combinations of the three groups revealed significant differences between control vs. Gmm ${ }^{\text {Wgm- }}$ males (Wilks' Lambda $\left.=0.253, \mathrm{X}^{2}=21.3, \mathrm{df}=5, p<0.001\right)$; control vs. $\mathrm{Gmm}^{\text {Apo }}$ males (Wilks' Lambda $=0.146, \mathrm{X}^{2}=29.8, \mathrm{df}=5$, $p<0.001$ ), but not between $\mathrm{Gmm}^{\text {Wgm- }}$ and $\mathrm{Gmm}^{\text {Apo }}$ males (Wilks' Lambda $=0.727, \mathrm{X}^{2}=4.9, \mathrm{df}=5, p<0.424$ ).

\section{Influence of antibiotic treatment in individually reared flies}

In individually reared 10-day-old females, there was no difference in the total amount of $\mathrm{CHCs}$ between control and $\mathrm{Gmm}^{\mathrm{Apo}}$ flies (Fig. 1a; t-test, $T=-0.888 \mathrm{df}=16, p=0.388$ ), nor in the absolute amount of female sex pheromone (Fig. 1c; t-test, $T=0.170, \mathrm{df}=16, p=0.868$ ). A comparison of the relative amounts of 15,19,23-trimethyl-heptatriacontane revealed significantly lower proportions of sex pheromone in $\mathrm{Gmm}^{\text {Apo }}$ females as compared to control flies (Fig. 1d; t-test, $T=2.080, \mathrm{df}=17, p=0.044$ ). Based on the 18 quantified peaks, four principal components were extracted, capturing $87.2 \%$ of the total variance. A discriminant analysis (DA) based on the four PCs including yielded a significant difference in $\mathrm{CHC}$ profiles between control and $\mathrm{Gmm}^{\text {Apo }}$ females (Fig. 2c; Wilks' Lambda $=0.233, \mathrm{X}^{2}=$ $21.8, \mathrm{df}=4, p<0.001)$. Based on the first discriminant functions, $94.7 \%$ of the cases were correctly classified $(50 \%$ would be expected by chance).

As in females, individually reared 10-day-old males showed no difference in the total amount of $\mathrm{CHCs}$ between control and $G m m^{\text {Apo }}$ flies (Fig. 1b; t-test for non-equal variances, $T=0.287, \mathrm{df}=11.653, p=0.779$ ). Based on the 13 quantified peaks, four principal components were extracted, capturing $87.2 \%$ of the total variance. A discriminant analysis (DA) based on the four PCs yielded a significant difference in $\mathrm{CHC}$ profiles across groups (Fig. 3c; Wilks' Lambda $=0.246, \mathrm{X}^{2}=18.2$, $\mathrm{df}=4, p=0.0011$ ). Based on one discriminant function, $94.1 \%$ of the cases were correctly classified (50\% would be expected by chance).

\section{Comparison of $\mathrm{CHC}$ profiles between antibiotic experiments}

A comparison of chemical profiles of $\mathrm{Gmm}^{\mathrm{Apo}}$ and control flies between the three experiments revealed significant differences between treatments and experiments for both females (Additional file 1a; Wilks' Lambda $=0.027, \mathrm{X}^{2}=$ 192.2, $\mathrm{df}=20, p<0.001$ ) and males (Additional file 1b; Wilks' Lambda $\left.=0.005, \mathrm{X}^{2}=250.6, \mathrm{df}=20, p<0.001\right)$. In particular, fly profiles were very well separated into the three experiments in the discriminant analysis.

\section{Influence of gamma-irradiaton on male flies}

In a full factorial model, no overall differences in total $\mathrm{CHC}$ amounts could be detected between treatment groups (ANOVA $\mathrm{F}_{11,108}=1.292, p=0.239$; time points: $\mathrm{F}_{2,108}=$ 3.577, $p=0.031$; irradiation dose: $\mathrm{F}_{3,108}=0.114, p=0.952$; interaction: $\mathrm{F}_{6,108}=1.119, p=0.356$; Fig. 4).

Based on the 16 quantified peaks and the 12 treatment groups, three principal components were extracted, capturing $80.0 \%$ of the total variance. A discriminant analysis (DA) based on the three PCs including all twelve treatment groups yielded a significant difference in $\mathrm{CHC}$ profiles across groups (Wilks' Lambda $=0.592, \mathrm{X}^{2}=58.5$, $\mathrm{df}=33, p=0.004$; Additional file 2), but only $28.3 \%$ of the cases were classified correctly based on both 


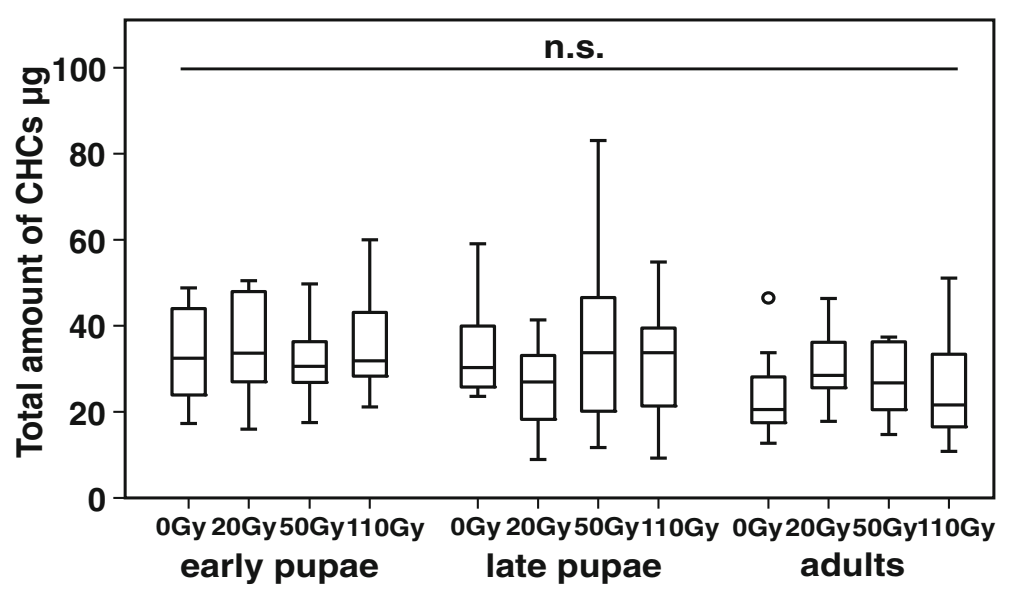

Fig. 4 Effect of gamma-irradiation on the total amount of CHCs in male Glossina m. morsitans flies. OGy= control (without irradiation). Male flies were irradiated at one of three different time points (early pupae, age 22 days; late pupae, 29 days; or young adults, 5 days). Lines represent medians, boxes comprise interquartile ranges, and whiskers denote minimum and maximum values, except for outliers that lie further away from a quartile than 1.5 times (circles) or 3 times (asterisks) the interquartile range

discriminant functions. When treatment time point was used as a grouping variable, the groups also differed significantly (Wilks' Lambda $=0.781, \mathrm{X}^{2}=28.7, \mathrm{df}=6, p<$ 0.001 ; Additional file 3), with $60 \%$ of the cases being classified correctly. Subsequent DAs of irradiation treatments at single time points revealed no significant difference between irradiation treatments for any of the three time points (including the 0Gy control; early: Wilks' Lambda $=0.677, \mathrm{X}^{2}=13.9, \mathrm{df}=9, p=0.127$; late: Wilks' Lambda $=0.693, \mathrm{X}^{2}=13.0, \mathrm{df}=6, p=0.126$; adult: Wilks' Lambda $=0.799, \mathrm{X}^{2}=8.0, \mathrm{df}=6, p=0.539$; Fig. 5a-c).

\section{Mate choice assays with mass-reared flies}

Out of the 30 male mate choice assays, the males chose females with their native microbiota in 20 cases and

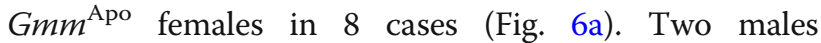

remained unmated. Excluding the unmated males, this distribution differs significantly from random mating ( $\mathrm{df}$ $=1, \mathrm{Chi}^{2}=5.14, p=0.02$ ). Of the 17 females, 10 mated with males with their native microbiota, two with $\mathrm{Gmm}^{\text {Apo }}$ males, and five remained unmated (Fig. 6b). Excluding the unmated females, this distribution differed significantly from random mating $\left(\mathrm{df}=1, \mathrm{Chi}^{2}=5.33, p=0.02\right)$.

\section{Discussion}

We assessed the impact of antibiotic treatment on the $\mathrm{CHC}$ profiles and mating success of male and female tsetse flies. Neither the absolute amount of all CHCs in females, nor the absolute amount of the female sex pheromone 15,19,23-trimethyl-heptatriacontane was effected by Amp or Tet treatment under any rearing condition. However, the relative amount of the sex pheromone was significantly
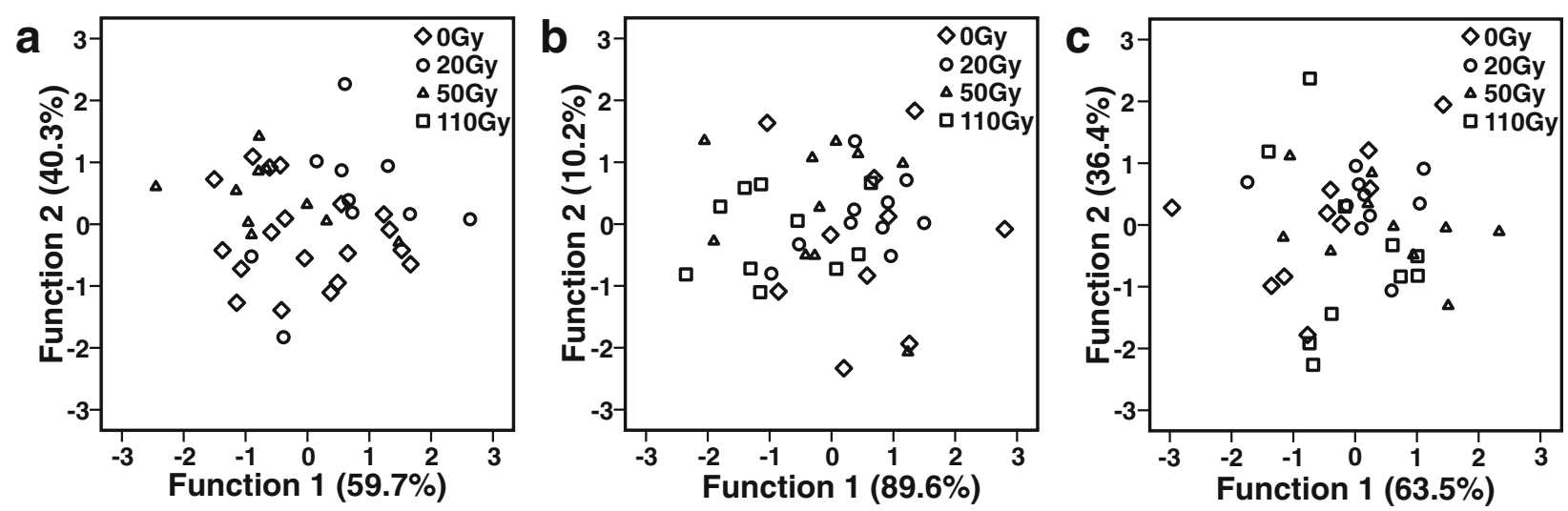

Fig. 5 Effect of gamma-irradiation dose on CHC profiles of male tsetse flies (G. m. morsitans). Discriminant analyses based on log-ratio transformed relative amounts of individually reared 10 day old males that were treated with different irradiation doses (0Gy, 20Gy, 50Gy, 110Gy) (a) during early pupal development (at day 22), (b) during late pupal development (after females emerged) and (c) to adult males (day 5 post-eclosion) 

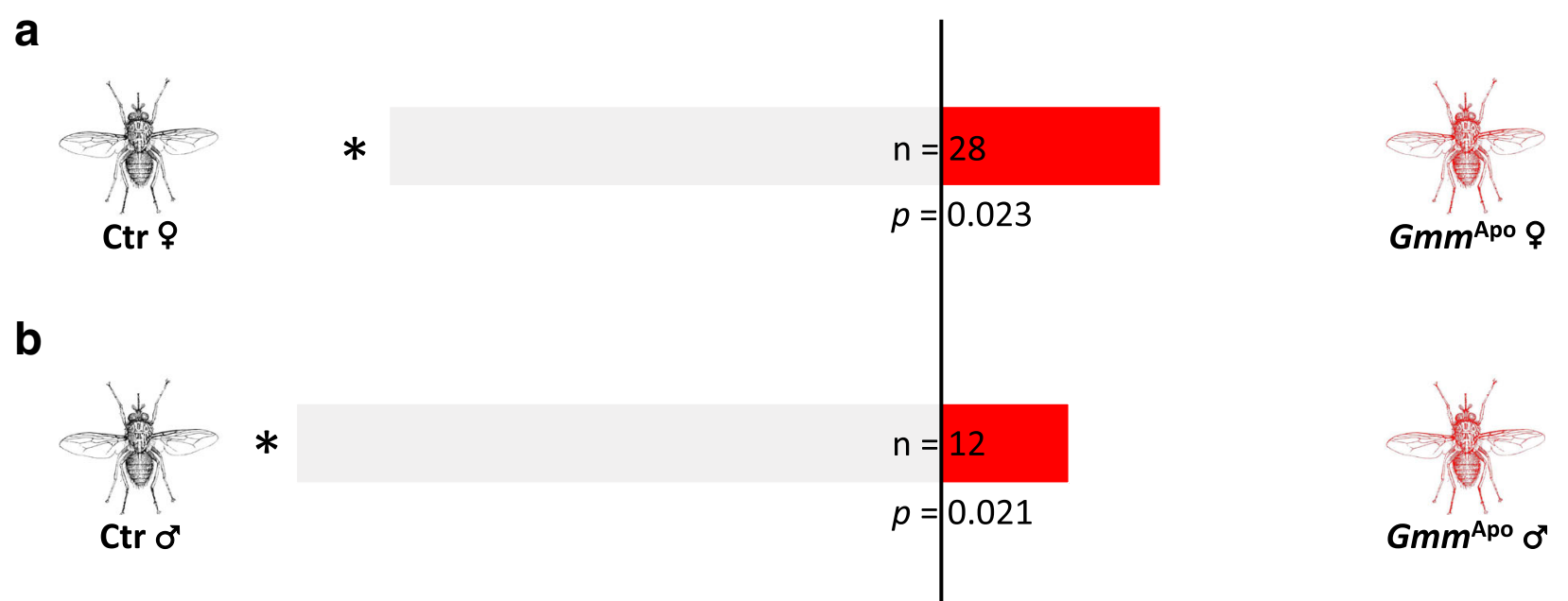

Fig. 6 Effect of tetracycline treatment on mating success of 5 day old (a) male and (b) female tsetse flies (G. m. morsitans). An untreated individual of the opposite sex was given a simultaneous choice between a $\mathrm{Gmm}^{\mathrm{Apo}}$ and an untreated individual. Ctr $=$ Control (without antibiotics), $\mathrm{Gmm}^{\mathrm{Apo}}=$ tetracycline-treated

reduced after Tet treatment. In males, the total amount of $\mathrm{CHCs}$ was significantly reduced in mass-reared $\mathrm{Gmm}^{\text {Apo }}$ males, but not in $\mathrm{Gmm}^{W g m-}$ and individually reared $\mathrm{Gmm}^{\text {Apo }}$ males. The $\mathrm{CHC}$ profiles of both females and males differed significantly between treatments under all rearing conditions except for mass reared $G m m^{W g m-}$ vs. $\mathrm{Gmm}^{\text {Apo }}$ 5-day old males. Further, gamma-irradiation of male pupae or young adults did not affect the $\mathrm{CHC}$ profile of 10-day old males, even though a previous study has shown that the treatment with 110Gy causes significant, yet variable effects on the three symbiont titers, based on irradiation time [40]. Finally, both male and female flies with their native microbiota discriminated against $\mathrm{Gmm}^{\text {Apo }}$ flies in mate choice assays.

The bacterial symbionts harbored by tsetse flies exhibit differential sensitivity to antibiotics and irradiation. Only Wigglesworthia is sensitive to both Amp and Tet [27, 38], whereas all three symbionts are affected by Tet [27]. Finally, irradiation significantly affects Sodalis and Wolbachia, but not Wigglesworthia titers [40]. Thus, our treatments include tsetse flies with their full microbiota (untreated controls of both antibiotic and irradiation experiments), flies with normal Sodalis and Wolbachia titers but without Wigglesworthia $\left(\mathrm{Gmm}^{\text {Wgm- }}\right.$ resulting from Amp treatment), flies with normal Wigglesworthia, but reduced Sodalis and Wolbachia titers (Gmm ${ }^{\text {Sod-Wlb- }}$ resulting from some of the irradiation treatments) and fully aposymbiotic flies $\left(\mathrm{Gmm}^{\mathrm{Apo}}\right.$, resulting from Tet treatment). Tet treatment, which clears all symbionts, had the strongest and most consistent effect on $\mathrm{CHC}$ profiles of males and females, as well as on the relative amount of the female sex pheromone. Furthermore, males mate preferentially with untreated females, possibly because their $\mathrm{CHC}$ profile contains a higher relative amount of 15,19,23-trimethyl-heptatriacontane, the female sex pheromone. Amp treatment also affected $\mathrm{CHC}$ profiles in males and 5-day old females, but not in 10-day old females, while irradiation which disturbs Sodalis and Wolbachia did not affect the CHC profiles of male flies. However, we cannot exclude the possibility that other time points of irradiation would yield different results, given the complex interaction effects of irradiation dosage and time on symbiont titers [40]. Nevertheless, taken together, these results suggest that Wigglesworthia has the strongest effect on $\mathrm{CHC}$ profiles of G. m. morsitans.

There is increasing evidence that symbiotic bacteria can under certain circumstances influence pheromone communication and mate choice of their insect host [7, 14-16, 43-49], which can ultimately result in reproductive isolation and, hence, speciation $[11,17,44]$. While reproductive manipulators like Wolbachia are prime suspects for the modification of their host's chemical communication and mate choice $[11,17,44]$, several gut associated microbes are also known to be involved in the production of host pheromone components. By contrast, nutritional endosymbionts like Wigglesworthia were so far not implicated in changes of host mating signals or mate choice. However, as Wigglesworthia provides essential vitamins [24] and is involved in the maturation of the immune system [25, 26, 27] direct or indirect effects on other metabolic processes such as the synthesis and distribution of hydrocarbons or their precursors seem plausible and could explain the modification of $\mathrm{CHC}$ profiles upon antibiotic treatment observed in our study.

Although our results are consistent with the hypothesis that an effect of Wigglesworthia on $\mathrm{CHC}$ profiles 
modulates mate choice, we could not test this effect on mate choice directly, nor is it currently possible to exclude direct effects of the antibiotic treatment itself on the fly's physiology, $\mathrm{CHC}$ profile, overall vigor, and behavior. Antibiotics influence several life history parameters of insects. For example, treatment of the black bean aphid (Aphis fabae; [48, 49]), the mustard aphid (Lipaphis erysimi; [50]), the walnut husk fly (Rhagoletis complete; [51]) and the melonfly (Dacus cucurbitae; [52]) with Tet derivatives in particular causes diverse side effects including reduced larval development rate, adult size, weight, reproduction and longevity. However, as all these aphids harbor the obligate intracellular mutualist Buchnera aphidicola [53], and the gut microbiota of diverse true fruit flies also has a significant influence on host fitness [54, 55], a direct influence of the tested antibiotics on host physiology cannot be differentiated from an indirect influence via symbiont depletion in these studies. A few studies have succeeded in implicating the insect microbiota in $\mathrm{CHC}$ profile modulation, without the involvement of antibiotics. Guo et al. [56] demonstrated that the gut microbiota of termites provides precursors for the synthesis of methyl-branched $\mathrm{CHCs}$ through the incorporation of ${ }^{14} \mathrm{C}$-labelled succinate. Furthermore, Dosmann et al. [57] investigated a possible microbial modulation of nest mate recognition in harvester ants by altering the external microbiome through antibiotic treatment, but also exclusively through the application of cultured bacteria to the ant cuticle. While the application of cultured microbes influenced nest mate recognition, treatment with rifampicin did not [57]. Thus, while direct contributions of obligate symbionts to nest mate recognition cues in harvester ants are possible, the results remain inconclusive.

Another factor that warrants careful interpretation of the presented results is that differences in $\mathrm{CHC}$ profiles across experiments were more pronounced than between treatments within each experiment (Additional file 1). Hence, the age of the flies, the rearing conditions (individual vs. mass-rearing), and possibly fluctuations in rearing conditions (e.g. diet, temperature, humidity) as well as variation in the genetic composition of the starting populations may influence $\mathrm{CHC}$ composition. Furthermore, the Sodalis and Wolbachia depleted flies resulting from gamma-irradiation were treated themselves, as opposed to analyzing the offspring of treated flies, as in the antibiotic experiments. Flies thus have experienced a different ontogeny, with Sodalis and Wolbachia present during part of their development. Despite the fact that cuticular hydrocarbons usually display a fast turnover, enabling insects to adapt within hours to days, it cannot be excluded that the late time point of symbiont depletion in the irradiation treatment was responsible for the lack of an effect on $\mathrm{CHC}$ profiles. Age and ontogeny-dependent changes in $\mathrm{CHCs}$ have been described across different insect species [58-62] and may serve as reliable age indicators for mate choice [63]. Diet and host genetics influence $\mathrm{CHCs}$ via fatty acid metabolism [64, 65], whereas fluctuations in temperature and humidity can stimulate insects to adjust their $\mathrm{CHC}$ profiles to improve desiccation resistance under the current conditions [66-68]. Thus, under natural settings, microbial symbionts may be one of several different factors affecting insect $\mathrm{CHC}$ profiles and thereby mate choice and sexual selection.

\section{Conclusion}

Our results provide first insights into changes in $\mathrm{CHC}$ profiles upon symbiont depletion by antibiotic and gamma-irradiation treatment in G. m. morsitans. Individual rearing corroborated the results obtained from mass-rearing, excluding potential pseudoreplication artifacts by flies exchanging $\mathrm{CHCs}$ through direct contact under mass-rearing conditions. Mate choice assays indicate that antibiotic treatment not only affects $\mathrm{CHC}$ composition, but also impairs mating success of both males and females. However, the link between mating success, $\mathrm{CHC}$ profiles, and Wigglesworthia as the causative agent for the observed changes remains speculative at this point. Further studies are needed to pinpoint single symbiont contributions to $\mathrm{CHC}$ synthesis and mate choice. Nevertheless, our results indicate that the chemical ecology of tsetse flies should be taken into account when investigating the effects of symbionts on host fitness or manipulating the symbiosis to enhance refractoriness to trypanosome infection. Furthermore, we could show that gamma-irradiation, which is routinely employed to create sterile males for the sterile-insect-technique to control G. m. morsitans populations, does not alter the $\mathrm{CHC}$ profiles of males. Hence, irradiated males might not suffer a competitive disadvantage after their release into the field, if females use chemical cues for mate choice. Finally, if symbiont or parasite infection predictably affects $\mathrm{CHC}$ profiles, chemical analyses may also provide a simple and cost-efficient alternative to molecular screenings for the assessment of symbiont/parasite infection status.

\section{Additional files}

Additional file 1: Comparison of $\mathrm{CHC}$ profiles of untreated $(\mathrm{Ctr})$ and tetracycline-treated (Tet) (a) female and (b) male tsetse flies (G. $m$. morsitans) across the three different experiments. (PDF $1363 \mathrm{~kb}$ )

Additional file 2: Effect of gamma-irradiation dose and time point on CHC profiles of 10 day old individually reared adult G. m. morsitans males. Discriminant analysis based on log-ratio transformed relative amounts across all treatment groups (time points and irradiation doses). (PDF $1353 \mathrm{~kb}$ ) 
Additional file 3: Effect of the time point of gamma-irradiation on $\mathrm{CHC}$ profiles of individually reared 10 day old adult G. m. morsitans males. Discriminant analysis based on log-ratio transformed relative amounts across irradiation time points (early and late pupal development and as young adults). (PDF $1354 \mathrm{~kb}$ )

\section{Abbreviations}

Amp: Ampicillin; ANOVA: Analysis of variance; Apo: Aposymbiotic; CHC: Cuticular hydrocarbon; DA: Discriminant analysis; FAO/IAEA: Joint food and agriculture organization and international atomic energy agency of the United Nations; Gmm: Glossina morsitans morsitans; Tet: Tetracyclin; Wgm: Wigglesworthia depleted

\section{Acknowledgements}

This project was part of the coordinated research project "Enhancing Vector Refractoriness to Trypanosome Infection" of the Joint FAO/IAEA Division of Nuclear Techniques in Food and Agriculture. We thank the members of CRP for fruitful discussions and feedback on the project.

\section{Funding}

Publication of this article was partially funded by the Max-Planck-Society (positions of TE and MK), as well as support from the Slovak Research and Development Agency under the contract No. APW-15-0604 entitled "Reduction of fecundity and trypanosomias control of tsetse flies by the application of sterile insect techniques and molecular methods" (to PT). Additional funding for the publication of this article was provided by the FAO/IAEA.

\section{Availability of data and materials}

Data presented in this article is available on figshare under doi:https:// doi.org/10.6084/m9.figshare.5472682 and doi: https://doi.org/10.6084/ m9.figshare.5473153.

\section{About this supplement}

This article has been published as part of BMC Microbiology Volume 18 Supplement 1, 2018: Enhancing Vector Refractoriness to Trypanosome Infection. The full contents of the supplement are available online at https:/ bmcmicrobiol.biomedcentral.com/articles/supplements/volume-18supplement-1.

\section{Authors' contributions}

WM, AA-A, SA and MK conceived the study, TE, VM, BW, GU, PT, WM, AA-A, SA and MK designed experiments, TE, VM, BW, GU, PT, MK performed experiments and analyzed the data, TE and MK wrote the initial manuscript, all authors commented on the manuscript, all authors read and approved the final manuscript.

\section{Ethics approval and consent to participate}

Not applicable

\section{Consent for publication}

Not applicable

\section{Competing interests}

The authors declare that they have no competing interests.

\section{Publisher's Note}

Springer Nature remains neutral with regard to jurisdictional claims in published maps and institutional affiliations.

\footnotetext{
Author details

'Insect Symbiosis Research Group, Max Planck Institute for Chemical Ecology, Jena, Germany. ${ }^{2}$ Department for Evolutionary Ecology, Institute for Organismic and Molecular Evolution, Johannes Gutenberg-University Mainz, Mainz, Germany. ${ }^{3}$ Department of Epidemiology of Microbial Diseases, Yale School of Public Health, New Haven, CT, USA. ${ }^{4}$ Institute of Zoology, Slovak Academy of Sciences, Bratislava, Slovakia. ${ }^{5}$ Present Address: Department of Biological Sciences, Florida International University, Miami, FL, USA. ${ }^{6}$ Insect Pest Control Laboratory, Joint FAO/IAEA Division of Nuclear Techniques in Food \& Agriculture, Vienna, Austria. ${ }^{7}$ Institute of Chemical, Environmental and Biological Engineering, Research Area Biochemical Technology, Vienna
}

University of Technology, Vienna, Austria. ${ }^{8}$ Laboratories of Genome Dynamics, Department Cell and Developmental Biology, Medical University of Vienna, Vienna, Austria.

Published: 23 November 2018

\section{References}

1. Blomquist GJ, Bagnères A-G. Insect hydrocarbons: biology, biochemistry, and chemical ecology. Cambridge: Cambridge University Press; 2010.

2. Howard RW, Blomquist GJ. Chemical ecology and biochemistry of insect hydrocarbons. Annu Rev Entomol. 1982;27:149-72.

3. Howard RW, Blomquist GJ. Ecological, behavioral, andbiochemical aspects of insect hydrocarbons. Annu Rev Entomol. 2005:50:371-93.

4. Le Conte Y, Hefetz A. Primer pheromones in social hymenoptera. Annu Rev Entomol. 2008:53:523-42.

5. Martin S, Drijfhout F. A review of ant Cuticular hydrocarbons. J Chem Ecol. 2009:35(10):1151-61

6. Carlson DA, Mayer MS, Silhacek DL, James JD, Beroza M, Bierl BA. Sex attractant pheromone of house Fly - isolation, identification and synthesis. Science. 1971;174(4004):76

7. Tobias Engl, Martin Kaltenpoth. Influence of microbial symbionts on insect pheromones. Natural Product Reports. 2018;35(5):386-397.

8. Douglas AE. The microbial dimension in insect nutritional ecology. Funct Ecol. 2009;23(1):38-47.

9. Feldhaar $\mathrm{H}$. Bacterial symbionts as mediators of ecologically important traits of insect hosts. Ecol Entomol. 2011:36(5):533-43.

10. Florez LV, Biedermann PHW, Engl T, Kaltenpoth M. Defensive symbioses of animals with prokaryotic and eukaryotic microorganisms. Nat Prod Rep. 2015;32(7):904-36.

11. Brucker RM, Bordenstein SR. Speciation by symbiosis. Trends Ecol Evol. 2012; 27(8):443-51.

12. Dillon RJ, Vennard CT, Charnley AK. A note: gut bacteria produce components of a locust cohesion pheromone. J Appl Microbiol. 2002;92(4): 759-63.

13. Dillon RJ, Vennard CT, Charnley AK. Pheromones - exploitation of gut bacteria in the locust. Nature. 2000;403(6772):851.

14. Sharon G, Segal D, Ringo JM, Hefetz A, Zilber-Rosenberg I, Rosenberg E. Commensal bacteria play a role in mating preference of Drosophila melanogaster. P Natl Acad Sci USA. 2010;107(46):20051-6.

15. Arbuthnott $D$, Levin TC, Promislow DEL. The impacts of Wolbachia and the microbiome on mate choice in Drosophila melanogaster. J Evol Biol. 2016; 29(2):461-8.

16. Leftwich PT, Clarke NVE, Hutchings Ml, Chapman T. Gut microbiomes and reproductive isolation in Drosophila. P Natl Acad Sci USA. 2017;114(48): 12767-72.

17. Miller WJ, Ehrman L, Schneider D. Infectious speciation revisited: impact of Symbiont-depletion on female fitness and mating behavior of Drosophila paulistorum. PLoS Pathog. 2010:6(12):e1001214.

18. Lindh JM, Lehane MJ. The tsetse fly Glossina fuscipes fuscipes (Diptera: Glossina) harbours a surprising diversity of bacteria other than symbionts. Anton Leeuw Int J G. 2011;99(3):711-20.

19. Geiger A, Fardeau ML, Njiokou F, Ollivier B. Glossina spp. gut bacterial flora and their putative role in fly-hosted trypanosome development. Front Cell Infect Mi. 2013;3:34.

20. Aksoy E, Telleria EL, Echodu R, Wu YN, Okedi LM, Weiss BL, Aksoy S, Caccone A. Analysis of multiple tsetse Fly populations in Uganda reveals limited diversity and species-specific gut microbiota. Appl Environ Microb. 2014;80(14):4301-12

21. Attardo GM, Lohs C, Heddi A, Alam UH, Yildirim S, Aksoy S. Analysis of Milk gland structure and function in Glossina morsitans: Milk protein production, symbiont populations and fecundity. J Insect Physiol. 2008;54(8):1236-42.

22. Benoit JB, Attardo GM, Baumann AA, Michalkova V, Aksoy S. Adenotrophic Viviparity in tsetse flies: potential for population control and as an insect model for lactation. Annu Rev Entomol. 2015;60:351-71.

23. Wang JW, Weiss BL, Aksoy S. Tsetse fly microbiota: form and function. Front Cell Infect Mi. 2013:3:69.

24. Akman L, Yamashita A, Watanabe H, Oshima K, Shiba T, Hattori M, Aksoy S. Genome sequence of the endocellular obligate symbiont of tsetse flies, Wigglesworthia glossinidia. Nat Genet. 2002;32(3):402-7.

25. Weiss BL, Wang JW, Aksoy S. Tsetse immune system maturation requires the presence of obligate Symbionts in larvae. PLoS Biol. 2011;9(5):e1000619. 
26. Weiss BL, Wang JW, Maltz MA, Wu YN, Aksoy S. Trypanosome infection establishment in the tsetse Fly gut is influenced by microbiome-regulated host immune barriers. PLoS Pathog. 2013;9(4):e1003318.

27. Alam U, Medlock J, Brelsfoard C, Pais R, Lohs C, Balmand S, Carnogursky J, Heddi A, Takac P, Galvani A, et al. Wolbachia Symbiont infections induce strong cytoplasmic incompatibility in the tsetse Fly Glossina morsitans. PLoS Pathog. 2011;7(12):e1002415.

28. Doudoumis V, Alam U, Aksoy E, Abd-Alla AMM, Tsiamis G, Brelsfoard C Aksoy S, Bourtzis K. Tsetse-Wolbachia symbiosis: comes of age and has great potential for pest and disease control. J Invertebr Pathol. 2013;112:S94-S103.

29. Schneider DI, Garschall KI, Parker AG, Abd-Alla AMM, Miller WJ. Global Wolbachia prevalence, titer fluctuations and their potential of causing cytoplasmic incompatibilities in tsetse flies and hybrids of Glossina morsitans subgroup species. J Invertebr Pathol. 2013;112:S104-15.

30. Nelson DR, Carlson DA. Cuticular hydrocarbons of the tsetse flies Glossina morsitans morsitans, Glossina austeni and Glossina pallidipes. Insect Biochem. 1986:16(2):403-16.

31. Nelson DR, Carlson DA, Fatland CL. Cuticular hydrocarbons of tsetse flies II. Glossina fuscipes fuscipes, Glossina palpalis palpalis, Glossina palpalis gambiensis, Glossina tachinoides, and Glossina brevipalpis. J Chem Ecol. 1988; 14(3):963-87.

32. Carlson DA, Langley PA, Huyton P. Sex-pheromone of tsetse-Fly - isolation, identification, and synthesis of contact aphrodisiacs. Science. 1978; 201(4357):750-3

33. Carlson DA, Nelson DR, Langley PA, Coates TW, Davis TL, Leegwatervanderlinden ME. Contact sex-pheromone in the tsetse-Fly Glossina-Pallidipes (Austen) identification and synthesis. J Chem Ecol. 1984; 10(3):429-50.

34. Langley PA, Pimley RW, Carlson DA. Sex recognition pheromone in tsetse Fly Glossina morsitans. Nature. 1975;254(5495):51-3.

35. Carlson DA, Mramba F, Sutton BD, Bernier UR, Geden CJ, Mori K. Sex pheromone of the tsetse species, Glossina austeni: isolation and identification of natural hydrocarbons, and bioassay of synthesized compounds. Med Vet Entomol. 2005;19(4):470-9.

36. Carlson DA, Offor II, El Messoussi S, Matsuyama K, Mori K, Jallon JM. Sex pheromone of Glossina tachinoides: isolation, identification, and synthesis. J Chem Ecol. 1998;24(9):1563-75.

37. Kroiss J, Svatos A, Kaltenpoth M. Rapid identification of insect Cuticular hydrocarbons using gas chromatography-ion-trap mass spectrometry. J Chem Ecol. 2011;37(4):420-7.

38. Pais R, Lohs C, Wu YN, Wang JW, Aksoy S. The obligate mutualist Wigglesworthia glossinidia influences reproduction, digestion, and immunity processes of its host, the tsetse fly. Appl Environ Microb. 2008;74(19):5965-74

39. Feldmann $U$. Guidelines for the rearing of tsetse flies using the membrane feeding technique. In: JPR O'-O, editor. Techniques of insect rearing for the development of integrated pest and vector management strategies. Nairobi: ICIPE Science Press; 1994. p. 449-71.

40. Uzel DG, Andrew G, Parker AG, DeVought L, van den Abbeele J, Vreyse MJB, Mach RL, Abd-Alla AMM: Combining paratransgenesis with SIT: Impact of ionizing radiation on the prevalence of Sodalis glossinidius. 2018; https://doi. org/10.1186/s12866-018-1283-8

41. Wall R, Langley PA. The mating-behavior of tsetse-flies (Glossina) - a review. Physiol Entomol. 1993;18(2):211-8.

42. Aitchison J. The statistical analysis of compositional data. London: Chapman \& Hall; 1986.

43. Koukou K, Pavlikaki H, Kilias G, Werren JH, Bourtzis K, Alahiotisi SN. Influence of antibiotic treatment and Wolbachia curing on sexual isolation among Drosophila melanogaster cage populations. Evolution. 2006;60(1):87-96.

44. Miller W, Schneider D. Endosymbiotic microbes as adaptive manipulators of arthropod behavior and natural driving sources of host speciation. In: Hughers DP, Brodeur J, Thomas F, editors. Host manipulation by parasites. Oxford: Oxford University Press; 2012.

45. Jacquet A, Horard B, Loppin B. Does pupal communication influence Wolbachia-mediated cytoplasmic incompatibility? Curr Biol. 2017;27(2):R53-5.

46. Pontier SM, Schweisguth F. A Wolbachia-sensitive communication between male and female pupae controls gamete compatibility in Drosophila. Curr Biol. 2015;25(18):2339-48

47. Pontier SM, Schweisguth F. Response to "does pupal communication influence Wolbachia-mediated cytoplasmic incompatibility?". Curr Biol. 2017; 27(2):R55-6
48. Jayaraj $S$, Ehrhardt $P$, Schmutterer $H$. The effect of certain antibiotics on reproduction of the black bean aphid, Aphis fabae Scop. Ann Appl Biol. 1967;59(1):13-21.

49. Lal OP. Wirkung einiger Antibiotika und Sulphonamide auf Fertilität, Lebensdauer, Gewicht und Größle von Aphis fabae Scop. Z Angew Entomol. 1971;68(1-4):158-63.

50. Lal OP. Effect of certain antibiotics on the development and Reproductivity of Lipaphis erysimi Kalt, on cabbage plant. Z Angew Entomol. 1972;70(1-4):82-8.

51. Tsiropoulos GJ. Effect of antibiotics incorporated into defined adult diets on survival and reproduction of the walnut husk fly, Rhagoletis completa cress. (Dipt., Trypetidae). Z Angew Entomol. 1981;91(1-5):100-6.

52. Sankaranarayanan R, Jayaraj S. Sterility-inducing effects of antibiotics and Sulphanilamide on the gourd fruitfly, Dacus cucurbitae coq. (Trypetidae, Diptera). Z Angew Entomol. 1975;79(1-4):129-36.

53. Douglas AE. Nutritional interactions in insect-microbial symbioses: aphids and their symbiotic bacteria Buchnera. Annu Rev Entomol. 1998;43:17-37.

54. Ben-Yosef M, Aharon Y, Jurkevitch E, Yuval B. Give us the tools and we will do the job: symbiotic bacteria affect olive fly fitness in a diet-dependent fashion. P Roy Soc B-Biol Sci. 2010;277(1687):1545-52.

55. Jurkevitch E. Riding the Trojan horse: combating pest insects with their own symbionts. Microb Biotechnol. 2011;4(5):620-7.

56. Guo L, Quilici DR, Chase J, Blomquist GJ. Gut tract microorganisms supply the precursors for methyl-branched hydrocarbon biosynthesis in the termite, Zootermopsis nevadensis. Insect Biochem. 1991;21(3):327-33.

57. Dosmann A, Bahet N, Gordon DM. Experimental modulation of external microbiome affects nestmate recognition in harvester ants (Pogonomyrmex barbatus). Peerj. 2016;4.

58. Howard RW, Howard CD, Colquhoun S. Ontogenic and environmentallyinduced changes in Cuticular hydrocarbons of Oryzaephilus surinamensis (Coleoptera, Cucujidae). Ann Entomol Soc Am. 1995;88(4):485-95.

59. Howard RW. Ontogenetic, reproductive, and nutritional effects on the cuticular hydrocarbons of the host-specific ectoparasitoid Cephalonomia tarsalis (Hymenoptera : Bethylidae). Ann Entomol Soc Am. 1998:91(1):101-12.

60. Tregenza T, Buckley SH, Pritchard VL, Butlin RK. Inter- and intrapopulation effects of sex and age on epicuticular composition of meadow grasshopper Chorthippus parallelus. J Chem Ecol. 2000;26(1):257-78.

61. Mpuru S, Blomquist GJ, Schal C, Roux M, Kuenzli M, Dusticier G, Clement JL Bagneres AG. Effect of age and sex on the production of internal and external hydrocarbons and pheromones in the housefly, Musca domestica. Insect Biochem Molec. 2001;31(9):935

62. Vanickova L, Svatos A, Kroiss J, Kaltenpoth M, Do Nascimento RR, Hoskovec M, Brizova R, Kalinova B. Cuticular hydrocarbons of the south American fruit Fly Anastrepha fraterculus: variability with sex and age. J Chem Ecol. 2012;38(9): 1133-42.

63. Kaltenpoth M, Strohm E. The scent of senescence: age-dependent changes in the composition of the cephalic gland secretion of the male European beewolf, Philanthus triangulum. J Insect Sci. 2006;6:20.

64. Blomquist GJ. Biosynthesis of cuticular hydrocarbons. In: Blomquist GJ Bagnères A-G, editors. Insect hydrocarbons: biology, biochemistry, and chemical ecology. Cambridge: Cambridge University Press; 2010. p. 35-52.

65. Wicker-Thomas C, Chertemps T. Molecular biology and genetics of hydrocarbon production. In: Blomquist GJ, Bagnères A-G, editors. Insect hydrocarbons: biology, biochemistry, and chemical ecology. Cambridge: Cambridge University Press; 2010. p. 53-74.

66. Gibbs AG, Rajpurohit S. Cuticular lipids and water balance. In: Bagnères A-G, Blomquist GJ, editors. Insect hydrocarbons: biology, biochemistry, and chemical ecology. Cambridge: Cambridge University Press; 2010. p. 100-20.

67. Chung H, Carroll SB. Wax, sex and the origin of species: dual roles of insect cuticular hydrocarbons in adaptation and mating. Bioessays. 2015;37(7):822-30.

68. Engl T, Eberl N, Gorse C, Krueger T, Schmidt THP, Plarre R, Adler C, Kaltenpoth M. Ancient symbiosis confers desiccation resistance to stored grain pest beetles. Mol Ecol. 2018;27(8):2095-2108. 